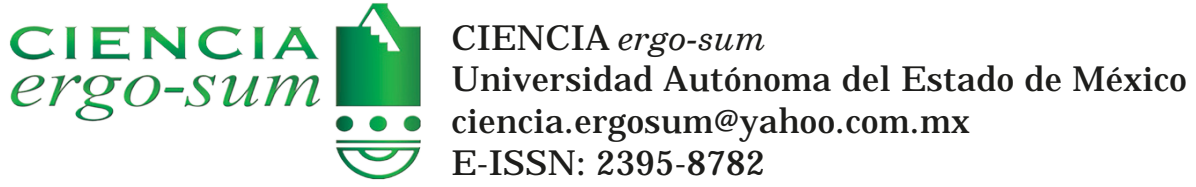

\title{
La prospectiva como marco de análisis para el aprovechamiento turístico de los hongos comestibles silvestres (HCS) en el centro de México
}

Gómez-Vázquez, Raúl; Thomé-Ortiz, Humberto; Pérez-Ramírez, Carlos Alberto y Martínez-García, Carlos Galdino

La prospectiva como marco de análisis para el aprovechamiento turístico de los hongos comestibles silvestres

(HCS) en el centro de México

CIENCIA ergo-sum, vol. 26, núm. 1, marzo-junio 2019|e44

Universidad Autónoma del Estado de México, México

Esta obra está bajo una Licencia Creative Commons Atribución-NoComercial-SinDerivar 4.0 Internacional.

Gómez-Vázquez, R., Thomé-Ortiz, H., Pérez-Ramírez, C, A. y Martínez-García, C. G. (2019). La prospectiva como marco de análisis para el aprovechamiento turístico de los hongos comestibles silvestres (HCS) en el centro de México. CIE NCIA ergo-sum, 26(1). https://doi.org/10.30878/ces.v26n1a9 


\section{La prospectiva como marco de análisis para el aprovechamiento turístico de los hongos comestibles silvestres (HCS) en el centro de México}

Prospective as a framework of analysis for the tourist use of Wild Edible Mushrooms (WEM) in the center of Mexico

Raúl Gómez-Vázquez

Universidad Autónoma del Estado de México, México

Recepción: 8 de febrero de 2018

r.g.vazquez91@gmail.com

Aprobación: 25 de junio de 2018

Humberto Thomé-Ortiz

Universidad Autónoma del Estado de México, México

humbertothome@hotmail.com

Carlos Alberto Pérez-Ramírez

Universidad Autónoma del Estado de México, México

capr.docencia@gmail.com

Carlos Galdino Martinez-García

Universidad Autónoma del Estado de México, México

cgmartinezg@uaemex.mx

\section{RESUMEN}

Los hongos comestibles silvestres (HCS) han sido aprovechados históricamente por las comunidades forestales, pues constituyen un componente fundamental de su cultura alimentaria y un recurso estratégico de subsistencia. Además, representan una oportunidad para la diversificación productiva local a partir del impulso del turismo rural orientado al micoturismo. Por este motivo, se delinea un marco de análisis teórico metodológico desde el enfoque de prospectiva. Como procedimiento metodológico se realizó una revisión de literatura del micoturismo y de los HCS en revistas científicas. Como resultado se identificaron 48 variables que inciden en la conformación del sistema micoturístico y que son una aproximación para generar escenarios futuros del aprovechamiento recreativo de los hongos comestibles silvestres.

Palabras Clave: micoturismo, prospectiva, variables.

\section{Abstract}

Wild Edible Fungi (WEF) have historically been used by forest communities as they are a fundamental component of their food culture and a strategic subsistence resource. In addition, they represent an opportunity for local productive diversification based on the impulse of rural tourism, oriented towards the impulse of mycotourism. The aim of the article article was to outline a framework of theoretical methodological analysis from a foresight perspective. As a methodological procedure, a review of the literature on mycotourism and the HCS was carried out in scientific journals. As a result, 48 variables were identified that affect the conformation of the mycotourism system and that are an approximation for the generation of future scenarios for the recreational use of wild edible fungi.

KEYWORDS: mycotourism, prospective, variables.

\section{INTRODUCCIÓN}

Las transformaciones socioeconómicas del espacio rural, en el contexto de la globalización, se han caracterizado por las dinámicas de poder en torno a cómo se reparten y distribuyen los recursos endógenos. 
El campo ha pasado de ser un proveedor de materias primas a una entidad biocultural estratégica para propiciar procesos de transformación socioeconómica. Por ello, a lo largo del siglo XX, la expansión de la industrialización ha incidido en que se masifiquen las producciones, el desplazamiento de los sistemas de producción tradicionales y los saberes locales (Gardner y Lewis, 2003).

En este contexto, las áreas forestales han sido objeto del uso irracional de sus recursos y la sobreexplotación, lo cual ha propiciado la pérdida de biodiversidad en los ecosistemas, la contaminación del suelo, de los mantos acuíferos y del aire, así como la marginación de los habitantes que dependían tanto de los recursos forestales no maderables (RFNM) como de los recursos forestales maderables (RFM).

A pesar de ello, los hongos comestibles silvestres (HCS) persisten como un importante recurso para los espacios forestales; lo anterior está asociado con las funciones que cumplen tanto para los ecosistemas como para la sociedad. Existen estudios sobre su importancia cultural, económica y biológica en países europeos, Estados Unidos, China y México (Esteban, 2007; Lázaro, 2008; Barron, 2011). En este sentido, el sector gubernamental de algunos países ha creado políticas específicas para la conservación y difusión de los hongos comestibles silvestres (Martínez et al., 2011a).

Recientemente, se ha impulsado el turismo micológico o micoturismo como una actividad a medio camino entre naturaleza y gastronomía. Esta vertiente promueve el desplazamiento del turista para disfrutar de estos recursos en su entorno natural; consta de tres componentes básicos: a) contacto con la naturaleza, b) degustación gastronómica y $c$ ) disfrute del patrimonio rural (Lázaro, 2008). Por su parte, Jiménez et al. (2016) lo definen como una modalidad híbrida de ocio turístico a partir de una oferta cultural étnica, ecológica y gastronómica en la que se funden diferentes motivaciones de viaje situadas en un punto intermedio entre naturaleza y cultura.

Este trabajo está soportado en una revisión de la literatura sobre aspectos teórico-metodológicos de los hongos comestibles silvestres y el micoturismo desde diferentes enfoques: económico, biológico, sociológico, cultural y político, la cual incluyó documentos publicados en el periodo 2005-2017 en revistas científicas. El documento se estructura en cuatro apartados. Primero, se retoman diversas nociones conceptuales sobre los recursos forestales no maderables y los hongos comestibles silvestres. Posteriormente, se describen los diversos abordajes teórico-metodológicos que se han empleado para el estudio del micoturismo; a partir de ello, se delinea un marco para su análisis. Por último, se realiza una discusión sobre la metodología propuesta de acuerdo con su pertinencia respecto a los estudios previos sobre el turismo micológico.

\section{Recursos forestales y hongos comestibles Silvestres (HCS)}

La simbiosis entre ser humano y naturaleza se asocia con los procesos de apropiación antrópica de los recursos naturales, donde el manejo de los bosques está vinculado con los conocimientos tradicionales de los grupos étnicos que han atravesado procesos de exclusión y marginación en el contexto de la globalización (Jasso et al., 2016). Los ecosistemas naturales proporcionan a la sociedad diversos servicios ambientales y recreativos, donde el bosque, debido a la diversidad recursos forestales maderables y de recursos forestales no maderables, ha fungido como catalizador de desarrollo para diferentes culturas. Dentro de los recursos forestales no maderables se encuentran los hongos, tanto alucinógenos como comestibles, que han sido parte de los rituales y de su dieta desde la época prehispánica, con mayor incidencia en la cultura mesoamericana (Martínez et al., 2011b).

Los hongos favorecen la estabilidad y productividad de los ecosistemas forestales al degradar moléculas orgánicas complejas en moléculas disponibles más simples en estado mineralizado, y además su consumo está asociado con el alto valor alimenticio que estos recursos proporcionan (Mariaca et al., 2001). Asimismo, son recursos de importancia socioeconómica para las comunidades forestales fungiendo como una fuente 
de ingresos y como parte de su cultura gastronómica. El uso de los recursos micológicos es significativo por razones ecocéntricas y antropocéntricas debido que durante la época de recolección representan una parte del ingreso parcial de quienes se dedican a esta actividad; de igual modo propician el vínculo intergeneracional de las familias inmersas en las áreas boscosas (Franco et al., 2012).

El aprovechamiento de los recursos micológicos incluye su uso directo por parte de las comunidades micofagas, por lo que es necesario considerar las implicaciones que subyacen al recolectarlos, ya que su alta extracción puede causar daño a la calidad del bosque, así como en la compactación del suelo y del bumus, con un efecto negativo en la producción de ciertas especies (Barron, 2011).

Por lo tanto, es necesario implementar políticas para regular los servicios proporcionados por el bosque con la finalidad de preservar los recursos de los cuales hacen uso tanto los habitantes locales como del exterior (Martínez et al., 2011a; Barron, 2011; Martínez, 2014), así como contribuir al diseño en conjunto con las comunidades locales de estrategias que permitan la preservación tanto los recursos forestales maderables como los recursos forestales no maderables.

Las especies micológicas se distribuyen a lo largo de todo un gradiente altitudinal que varia desde los 3000 y 3500 msnm en el que se incluyen diversas comunidades vegetales, zonas agrícolas y comunidades urbanas, lo cual refleja su capacidad adaptativa a los cambios de su ambiente natural (Franco et al., 2012). Debido a su distribución geográfica, la producción de hongos se relaciona con características ecológicas como la temperatura media mensual, la precipitación mensual, el diámetro y la edad promedio del arbolado, las cuales son variables que tienen mayor influencia en la producción de hongos en los bosques de Pinus hartwegii Lindl. y Abies religiosa (HBK) Schtdl. et Cham. (Fernández et al., 2012; Barron, 2011; Toledo et al., 2014).

Asimismo, los hongos comestibles silvestres desempeñan un papel importante entre los pobladores de Mesoamérica, principalmente en las tierras cubiertas de pino y encino. En México la recolección de hongos se encuentra ligada de manera muy estrecha con el conocimiento que poseen los habitantes de las comunidades campesinas, quienes en su mayoría han sido los encargados de transmitirlos de generación en generación, y facilita que se identifiquen las especies comestibles (Franco et al., 2012).

Para la recolección de estos hongos las personas deben conocer el entorno, las características de los lugares, así como los parajes boscosos. La actividad es llevada a cabo por toda la familia, y las madres y las abuelas son quienes más trasmiten el conocimiento en el núcleo familiar (Lara et al., 2013; Moreno, 2013; Burrola et al., 2012; Mariaca et al., 2001).

La recolecta, consumo y comercialización de hongos no es una actividad reciente, pero sí constituye un mecanismo para obtener alimentos silvestres, el cual es propio de las poblaciones establecidas en zonas boscosas a lo largo del proceso histórico; también involucra a la familia como unidad de producción y reproduce un profundo conocimiento ecológico tradicional integrado por distintos grupos étnicos acerca de los hongos comestibles silvestres. Sin embargo, se trata de un recurso al que los recolectores no pueden asignar un claro valor de mercado, agregar valor o diversificar su aprovechamiento en aras de construir relaciones de intercambio económico más equitativas debido a que es un producto obtenido del bosque y que relativamente no genera costos (Charnley et al., 2007).

\section{Abordajes teórico-Metodológicos del MiCoturismo}

Los trabajos relacionados con el micoturismo o turismo micológico se enfocan en la utilidad marginal de la recolección de hongos referentes al conjunto de bienes y servicios consumidos por los turistas, las dimensiones comercial y recreativa, el desarrollo sustentable, el etnoconocimiento, la interacción social, los aspectos biológicos y antropológicos, en los cuales se retoman puntos esenciales que aportaron a la identificación de las variables clave, así como en la coadunación de la propuesta metodológica. 
Actualmente, el alto valor de los hongos silvestres en los mercados nacionales e internacionales ha provocado una alta presión extractiva, lo que ha transformado su aprovechamiento y comercialización de un nivel de autoconsumo y venta local a la comercialización a gran escala. Lo anterior ha generado que se perturbe de manera excesiva el hábitat, lo que conlleva a que se degrade el suelo, a la eliminación de plantas y animales que interactúan con los hongos, se interrumpa su ciclo biológico y, por tanto, disminuya y fluctué la abundancia y la distribución de las principales especies de hongos. Además, la sobreexplotación puede interferir en el importante papel que juegan en el reciclamiento de nutrimentos y en la incorporación de materia orgánica al suelo (Alvarado y Benítez, 2009).

Asimismo, existe un acuerdo generalizado entre los micólogos acerca de que la disminución de las poblaciones de hongos puede tener repercusiones tanto para las comunidades humanas como no humanas. No obstante, se pueden plantear diferentes estrategias para mitigar dichos efectos, lo cual constituye una investigación útil para el manejo de los recursos (Barron, 2011).

En lo que respecta a México, en el área micoturística faltan tecnologías para el escalamiento de la producción rural de hongos, pues predomina una comercialización centralizada, la escasez de estadísticas y los estudios de mercado (nacional e internacional), escaso interés público y privado para incentivar la producción y el mercado, falta de legislación, problemas técnicos de almacenamiento, conservación y transporte.

Por ello, se debe garantizar la sustentabilidad de su práctica desde el punto de vista económico, social y ambiental, fomentar la combinación con otros recursos territoriales, promover la profesionalización del sector, potenciar sinergias entre los diferentes sectores implicados y fomentar actividades con mayor repercusión para que las comunidades puedan ofrecer un micoturismo de calidad, además de innovar y poner en marcha proyectos piloto; así pues, resulta necesario establecer mecanismos de seguimiento y la mejora del complejo modelo de gestión implantado, lo que permitiría conformar una oferta turística adecuada a la demanda en un mercado tan complejo como el del turismo rural (Martínez et al., 2011a).

De esta forma, desde una perspectiva económica para la diversificación productiva, De Frutos et al. (2011) reconocen la importancia de que el Estado intervenga para impulsar la conservación del recurso forestal y la gestión de infraestructura que puedan colocar a la actividad micoturística en un mejor escenario. Del mismo modo, Thomé (2016) propone el desarrollo de un centro de interpretación micológica, la creación de una marca micológica, la fabricación de paradores micogastronómicos de temporada y puntos de venta de productos micológicos, el desarrollo de una agroindustria rural especializada en la transformación de productos micológicos, así como de una plataforma de eventos para difundir la cultura micológica.

Por su parte, Carvalho (2009) plantea que se elabore un sistema básico de información micológica, una estrategia de gestión micoturística consistente en la planificación turística participativa, la zonificación de los senderos micológicos, regular la recolección recreativa de hongos comestibles silvestres y además un dispositivo de comunicación que permita difundir la cultura micológica, la gastronomía local y la importancia ecológica a partir de la vinculación entre el turismo y la educación ambiental.

Recolectar hongos es una actividad profundamente arraigada en la cultura de las comunidades indígenas y campesinas, las cuales han generado, reelaborado y transmitido su conocimiento de una generación a otra, lo que ha dado origen a sistemas de producción que han demostrado ser compatibles con la conservación a largo plazo.

Los hongos comestibles silvestres son importantes tanto para regular el ecosistema como para los recolectores que habitan en las áreas boscosas. La sustentabilidad de la actividad micoturística sólo será posible a través de estrategias encaminadas a fortalecer la capacidad técnica y organizativa de los poseedores de los recursos naturales, y así dar lugar a la revaloración del bosque. Esta circunstancia presenta oportunidades y retos que deben ser atendidos, por lo que se propone tanto la intervención científica como la aplicación de nuevos enfoques para lograr un equilibrio entre las necesidades del ser humano y la protección y conservación de los recursos naturales (Alvarado y Benítez, 2009). 
De este modo, un acercamiento al abordaje holístico para la actividad micoturística es el enfoque de los agroecosistemas, el cual permite destacar los beneficios que favorecen el balance ambiental, fortalecer la sustentabilidad, preservar el conocimiento empírico y ayudar al desarrollo de una legislación pertinente para la gestión del recurso. Así, la sustentabilidad no sólo dependerá de las características biológicas de una especie en particular sino del contexto socioeconómico, legal y cultural que lo enmarca. En este sentido, la intervención científica puede mejorar la capacidad de negociación y las técnicas de manejo en sistemas agroforestales con una mayor rentabilidad económica y equidad social (Alvarado y Benítez, 2009).

Por ello, el trabajo etnomicológico de Moreno (2002), el etnográfico de Butnaru (2015) y las aportaciones de Konu (2015) destacan la importancia del saber tradicional y las manifestaciones e implicaciones culturales y ambientales que se derivan de las relaciones establecidas entre los hongos y el ser humano; la identificación de los recolectores de la comunidad, el número de especies de hongos comestibles silvestres, los espacios de recolección y de venta, así como los precios, son los elementos centrales para construir una matriz sobre los impactos socioeconómicos generados por los usos actuales de los hongos comestibles silvestres.

Para poder construir una visión holística de la actividad del micoturismo, proponemos un acercamiento a dos aproximaciones teóricas que apoyen en la investigación y explicación del fenómeno turístico en las áreas forestales y también de su relación con los hongos comestibles silvestres. Destacan los siguientes estudios:

a) Agroecosistémica: esta aproximación teórica analiza los hongos comestibles silvestres como un sistema que está integrado por diferentes variables y categorías (Alvarado y Benítez, 2009).zxb) Prospectiva: se utiliza para el análisis de las cuestiones económicas, políticas, sociales y culturales de los hongos comestibles silvestres, así como la construcción de los escenarios para la actividad turística de manera sustentable (Carvalho, 2009; Salas, 2013; Thomé, 2016).

Sin embargo, a pesar de estas aportaciones, Mariaca et al. (2001) mencionan que hay una carencia de estudios referentes a la importancia que tienen los hongos comestibles para los grupos étnicos que habitan en regiones montañosas con un clima templado-húmedo o subhúmedo. Con esto, se muestra que se tiene aún mucho camino por recorrer en relación con el aprovechamiento de los hongos comestibles silvestres y la actividad turística al posicionar limitantes y problemáticas que pueden ser contrarrestadas como lo mencionan Alvarado y Benítez (2009), quienes realizan un estudio de forma integral. En ese sentido, se busca delinear los escenarios de la actividad micoturística en el centro de México y el diseño de un modelo metodológico para tal propósito.

\section{Prospectiva como herramienta metodológica para el abordaje integral del MICOTURISMO}

La prospectiva es una herramienta metodológica que sirve para construir diferentes escenarios de problemas específicos que van desde lo catastrófico hasta lo utópico, los cuales deben ser planteados a través del análisis del territorio seleccionando las variables mediante un panel de expertos. El concepto de prospectiva cobra importancia cuando se emplea para abordar el futuro económico y social de las regiones, el cual debe de reconocer las dimensiones del desarrollo local y las variables generadas por la sinergia de los actores en un territorio, y así encontrar las variables estratégicas sobre las cuales se deberían cimentar los planes de desarrollo local.

Burbano y Moreno (2015) reconocen que el análisis del futuro de un territorio resulta del esfuerzo de concentración y planificación emprendido por el conjunto de actores locales con el fin de valorizar los recursos humanos y materiales y mantener una negociación con los centros donde se toman decisiones económicas, 
sociales y políticas; de esta forma, la prospectiva en una localidad puede considerarse factible y efectiva, lo que facilitaría la planificación estratégica para alcanzar el futuro deseado.

La construcción de escenarios debe tomar en cuenta primero una reflexión en torno a variables clave y hechos portadores de futuro, seguida de las tendencias dentro del territorio, después el juego de actores y finalmente el planteamiento de supuestos. Su diseño requiere que se integre toda la información recopilada con el propósito de mantener el nivel de detalle manejado hasta el momento y describir no sólo los cambios que pudieran suscitarse en el territorio bajo estudio sino también los procesos por medio de los cuales tendrán lugar (Salas, 2013).

Así, para construir los escenarios de la actividad micoturística se tienen que considerar a los actores involucrados como lo son los recolectores, intermediarios, el gobierno local, la academia y los consumidores/ turistas con la finalidad de dimensionar apropiadamente las problemáticas sociales y económicas que existen en los espacios rurales donde se recolectan, consumen y distribuyen hongos comestibles silvestres. De acuerdo con lo anterior, es preciso reconocer que la prospectiva no es producto del azar, su conducción y consecución deben estar orientadas por una directriz clara sobre qué hacer y cómo mediante la participación directa de los actores locales.

Además, es necesario describir los progresivos cambios que se vayan experimentando dentro de los procesos socioterritoriales para delinear un conjunto de escenarios de los cuales se seleccionará aquel que la comunidad involucrada y los expertos consideren el de mayor posibilidad de concreción y el de mayor concordancia con los intereses colectivos. Las técnicas de prospección aportan una visión más amplia del desarrollo rural y la posibilidad de tener en cuenta los datos cualitativos y las estrategias desplegadas por los actores sociales implicados (Ortega, 2008).

Godet (2000) señala que el futuro no está determinado en su totalidad, puesto que en éste influyen actores con proyectos individuales y colectivos juegos de poder, intereses y necesidades cambiantes, de allí que no sólo se pronostique a partir de tendencias e hipótesis sobre su comportamiento sino que igualmente puede ser controlado y dirigido. En el ámbito del micoturismo, las hipótesis a plantear por variable clave deberán reconocer los cambios que incidirán en la apropiación, funcionalización, fragmentación, polarización y segregación o marginación espacial tomando en cuenta la influencia de los actores en esos procesos. Con este planteamiento, se pretende analizar el futuro de la actividad micoturística con el propósito de comprender las implicaciones culturales, sociales, económicas y políticas que puede generar dicha actividad.

A partir de las aportaciones previas, es posible construir los escenarios futuros de la actividad micoturística en el centro de México que retomen la metodología de prospectiva como marco de análisis, el cual se divide en dos fases:

a) La primera consiste en la construcción de la base analítica e histórica del sistema micoturístico en la que se identifican las variables clave mediante una revisión de literatura, se construye una matriz de impactos cruzados que es ponderada por un panel de expertos y se utiliza el software MICMAC para determinar la situación actual de la actividad, así como los gérmenes de cambio.

b) La segunda es mediante el análisis de juego de los actores para establecer hipótesis que vuelven a ser evaluadas por el panel de expertos. Una vez obtenidos los resultados, se validan con los software Mactor y Prob-Expert, donde se realiza un juego de hipótesis probabilizadas sobre las cuestiones clave para el futuro de la actividad micoturística. Posterior a ello, se procede a un análisis morfológico en el cual se construyen los escenarios de progresiones, imágenes y previsiones con el software Morphol: el método multicriterio para determinar las estrategias, alternativas y el plan de acciones con el fin de favorecer el aprovechamiento turístico de los hongos comestibles silvestres aunado a la actividad turística en una área forestal del centro de México (figura 1). 


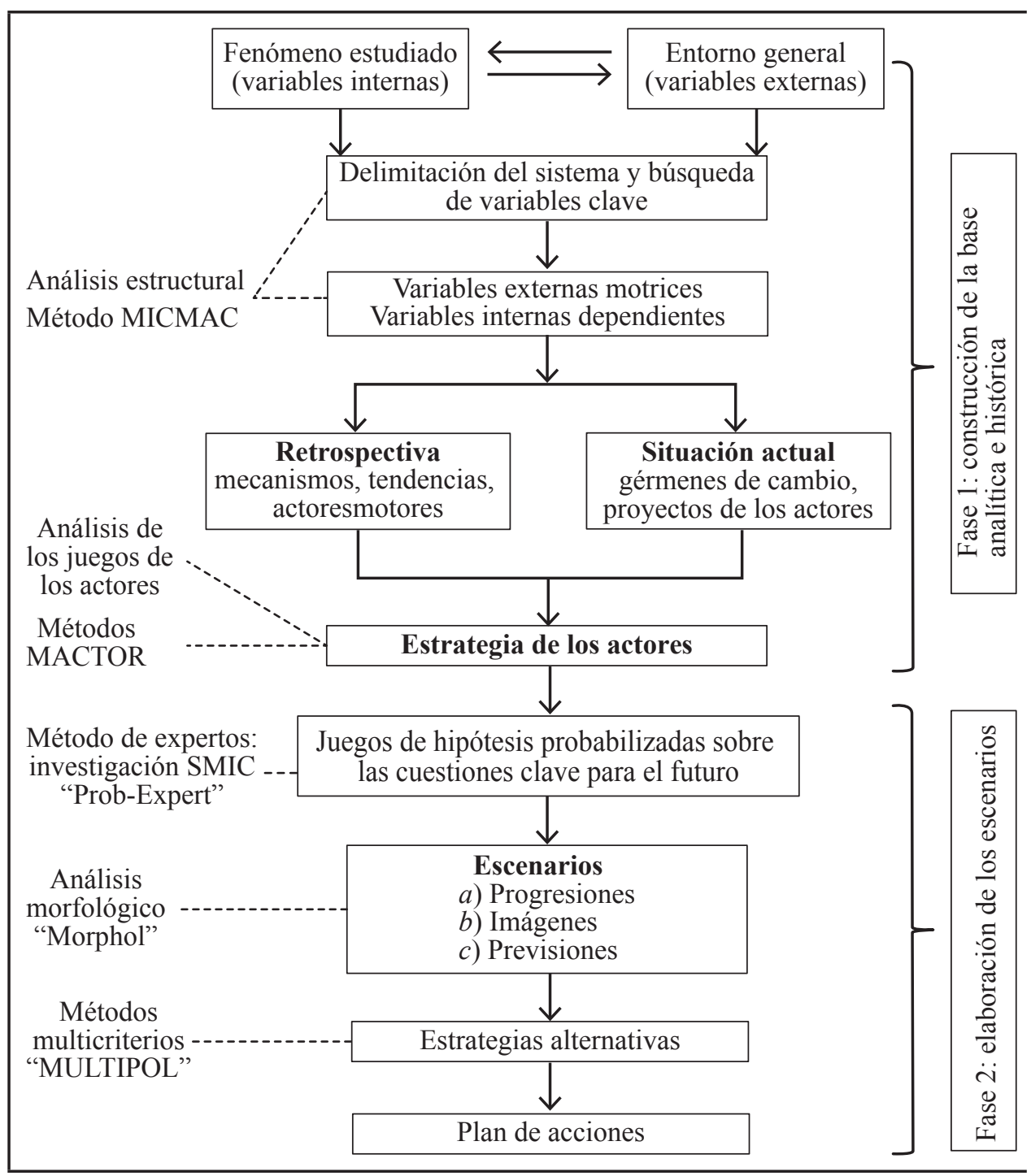

FIGURA 1

Procedimiento metodológico para la elaboración de escenarios de la actividad micoturística Fuente: elaboración propia a partir de Godet, 2000; Salas, 2013; Sarría y Becerra, 2008.

\section{1. Variables clave del sistema micoturístico}

Para obtener las variables clave se realizó un análisis de contenido en las aportaciones de investigaciones previas en el periodo de 2005 a 2017 relacionadas con hongos comestibles silvestres y micoturismo. Para la selección de las variables se empleó el método de moda (Mo) en los apartados de conclusiones, discusiones y proyecciones para el análisis integral del sistema. El análisis de contenido se efectuó en 16 artículos de revistas indexadas como Botánica, Estudios de Economía Aplicada, Anales de Geografía, Periplo Sustentable, Bosque, CIENCIA ergo-sum, Tropical and Subtropical Agroecosystems, Agricultura, Sociedad y Desarrollo, Revista Chapingo Serie Ciencias Forestales y del Ambiente, Revista Mexicana de Micología, Revista Iberoamericana de Micología, Fungal Ecology Forest Policy and Economics y Forest Ecology and Management.

Para poder construir el modelo metodológico de prospectiva de la actividad micoturística en las áreas forestales, se identificaron 48 variables clasificadas en ocho dimensiones que intervienen en la presencia y la calendarización de especies de hongos comestibles silvestres, la importancia socioeconómica que representan para los recolectores, la forma de tenencia de la tierra y su intervención dentro de las áreas forestales, así como en la intervención de 
diversos actores sociales en la actividad micoturística. Para la construcción de los escenarios futuros de la actividad micoturística, el cuadro 1 muestra las variables obtenidas a partir de la revisión de literatura desde diferentes enfoques mediante la exposición de las problemáticas, ventajas comparativas, cualidades ambientales y del entorno y de la importancia del conocimiento tradicional ecológico que ha sido heredado generacionalmente entre los recolectores.

\section{CUADRO 1}

Variables clave del sistema micoturístico

\begin{tabular}{|c|c|}
\hline Dimensión & Variable clave \\
\hline \multirow{12}{*}{$\begin{array}{l}\text { 1. Características biofísicas } \\
\text { (Mariaca et al., 2001; Burrola } \\
\text { et al., 2012; Aguirre et al., } \\
\text { 2013; Lara et al., 2013; } \\
\text { Martínez, 2014; Jasso et al., } \\
\text { 2016). }\end{array}$} & Precipitaciones forestales \\
\hline & Cambio climático \\
\hline & Densidad forestal \\
\hline & Fructificación de hongos \\
\hline & Deterioro ambiental \\
\hline & Función en los ecosistemas \\
\hline & Estabilidad de los ecosistemas \\
\hline & Productividad de los ecosistemas \\
\hline & Sequía \\
\hline & Falta de humedad \\
\hline & Plagas \\
\hline & Disponibilidad de recursos naturales \\
\hline \multirow{8}{*}{$\begin{array}{l}\text { 2. Calendarización de la } \\
\text { fructificación (Martínez, 2014; } \\
\text { Martínez et al., 2011b). }\end{array}$} & Posibilidad de aprovechamiento de recursos naturales \\
\hline & Altitud sobre el nivel del mar \\
\hline & Distribución ecológica \\
\hline & Distribución geográfica \\
\hline & Tipos de vegetación que intervienen en el ciclo de los hongos comestibles silvestres \\
\hline & Estacionalidad de los hongos comestibles silvestres \\
\hline & Externalidades forestales \\
\hline & Disminución de materia orgánica \\
\hline \multirow{10}{*}{$\begin{array}{l}\text { 1. Importancia socioeconómica } \\
\text { de las especies (Jiménez et al., } \\
\text { 2016; Zizumbo et al., 2012; } \\
\text { Barron, 2011). }\end{array}$} & Crecimiento de la población \\
\hline & Tala de árboles \\
\hline & Actividad extractiva de los recursos forestales no maderables \\
\hline & Capacidad de uso de la tierra \\
\hline & Cambio de uso del suelo \\
\hline & El pastoreo \\
\hline & Conflictos de uso de la tierra \\
\hline & Comercialización de los hongos comestibles silvestres \\
\hline & Ingresos obtenidos de la recolección de hongos comestibles silvestres \\
\hline & Variabilidad de los precios de los hongos comestibles silvestres \\
\hline \multirow{6}{*}{$\begin{array}{l}\text { 2. Formas de tenencia de la } \\
\text { tierra (Fernández et al., 2012). }\end{array}$} & Intervención de la mujer en la recolección \\
\hline & Fortalecimiento de los vínculos intergeneracionales \\
\hline & Sentido de identidad que brinda la recolección de hongos comestibles silvestres \\
\hline & Conocimiento tradicional \\
\hline & Conocimiento del entorno \\
\hline & Creencias místicas \\
\hline \multirow{5}{*}{$\begin{array}{l}\text { 3. Mercados regionales y } \\
\text { locales de hongos comestibles } \\
\text { silvestres (Thomé, 2016; } \\
\text { Lázaro, 2008; De Frutos et al., } \\
\text { 2011). }\end{array}$} & Autoconsumo \\
\hline & Transformación de los hongos comestibles silvestres en un producto gastronómico \\
\hline & Empleos a partir del micoturismo \\
\hline & Incremento de turistas \\
\hline & Paradores micoturísticos \\
\hline \multirow{6}{*}{$\begin{array}{l}\text { 1. Intervención de la academia } \\
\text { 2. Intervención del Estado } \\
\text { 3. Intervención de ONG } \\
\text { (Alvarado y Benítez, 2009; } \\
\text { Arteaga y Moreno, 2006) }\end{array}$} & Incremento de los intereses en la conservación ambiental \\
\hline & Implementación de políticas públicas \\
\hline & Organización institucional para la ordenación del territorio \\
\hline & Financiamiento para el funcionamiento institucional \\
\hline & Personal capacitado y comprometido \\
\hline & Alianzas estratégicas institucionales \\
\hline
\end{tabular}

Fuente: elaboración propia. 
Las variables presentadas en el cuadro 1 son un bosquejo en el que se exponen las limitantes y avances relacionados con la recolección, venta e intercambio que existen en las áreas rurales, las cuales cuentan con las características territoriales que permiten la fructificación de hongos comestibles silvestres. Asimismo, se presentan las oportunidades al incurrir con la actividad del micoturismo para el aprovechamiento sustentable de los hongos y a la vez generar ingresos económicos que repercutan de una manera positiva, además de crear un anclaje territorial de los habitantes de las áreas boscosas a través del conocimiento tradicional ecológico que poseen.

La prospectiva es un marco de análisis en el que se plasma una visión multidisciplinaria para el abordaje de la actividad micoturística. La intervención de organismos locales y estatales repercuten en el incremento del interés por la conservación de ambiental mediante políticas públicas o la organización institucional para la ordenación del territorio, lo que posibilita crear alianzas estratégicas institucionales.

\section{Conclusiones}

El micoturismo es una actividad que se encuentra en una fase de germinación en las áreas forestales. Es necesario determinar nuevos modelos de planificación turística encaminados a fortalecer las comunidades receptoras y a su vez involucrar a la mayoría de los actores locales insertos dentro de dicha actividad para la generación de un anclaje territorial; por ello, la prospectiva es un modelo que, además de incluir a los actores locales e institucionales, se complementa con el soporte del conocimiento local y el científico desde diferentes disciplinas para construir escenarios que determinen la viabilidad del micoturismo.

La creación de una perspectiva integral del micoturismo para el aprovechamiento de los hongos comestibles silvestres se logró a través de la revisión de literatura, pues a partir de ella se pudo delinear un marco de análisis con base en las orientaciones metodológicas. Las variables presentadas son un primer acercamiento para la construcción de escenarios futuros de la actividad micoturística en un área forestal del centro de México que cuenta con las características territoriales y climatológicas para la proliferación de hongos.

A partir de la revisión de las orientaciones metodológicas de los hongos comestibles silvestres fue posible delinear una propuesta del análisis con enfoque multidisciplinario y prospectivo que pretenda contribuir a la construcción de los escenarios para el desarrollo de la actividad micoturística, de tal manera que sean discutidos por la comunidad local y académica desde el enfoque de la prospectiva. La obtención de las primeras variables y la validación en conjunto con el panel de expertos permite consolidar los escenarios conforme a la experiencia de los integrantes desde la ciencia y el conocimiento del entorno, así como de las prácticas de recolección de hongos comestibles silvestres que podrán fortalecer la actividad micoturística en el área de estudio.

\section{Agradecimientos}

$\mathrm{Al}$ apoyo del proyecto "Evaluación de la dimensión recreativa de los hongos comestibles silvestres, su interés socioeconómico y sus perspectivas de desarrollo rural” financiado por CONACYT-SEP Ciencia Básica 2014.

\section{REFERENCIAS}

Aguirre, J. L., Díaz, G., Larrán, A., Aparicio, A., Talabante, C. y Rodríguez, A. (2013). Estudio del potencial micológico en las zonas rurales de Guadalajara en relación con su aprovechamiento turístico. Guadalajara: FSE.

Alvarado, G. y Benítez, G. (2009). El enfoque de agroecosistemas como una forma de intervención científica en la recolección de hongos silvestres comestibles. Tropical and Subtropical Agroecosystems, 10(3), 531-539. 
Arteaga, B. y Moreno, C. (2006). Los hongos comestibles silvestres de Santa Catarina del Monte, Estado de México. Revista Chapingo. Serie Ciencias Forestales y del Ambiente, 12(2), 125-131.

Barron, E. (2011). The emergence and coalescence of fungal conservation social networks in Europe and the U.S.A. Fungal Ecology, 4, 124-133. https://doi.org/10.1016/j.funeco.2010.09.009.

Burbano, E. L. y Moreno, E. (2015). El desarrollo local desde una visión de prospectiva. Revista Virtual Universidad Católica del Norte, 45, 245-257.

Butnaru, G. I. (2015). The method of ethnographic and content analysis in determining development factors of economic and managerial tourism performance. Procedia Economics and Finance, 20, 104-11. https://doi.org/10.1016/S2212-5671(15)00053-2.

Burrola, C., Montiel, O., Garibay, R. y Zizumbo, L. (2012). Conocimiento tradicional y aprovechamiento de los hongos comestibles silvestres en la región de Amanalco, Estado de México. Revista Mexicana de Micologia, 35, 1-16.

Carvalho, S. M. (2009). Micoturismo: enquadramento estratégico em áreas protegidas. Lisboa, Portugal.

Charnley, S., Fischer, A. P. y Jones, E. T. (2007). Integrating traditional and local ecological knowledge into forest biodiversity conservation in the Pacific Northwest. Forest Ecology and Management, 246, 14-28. https://doi.org/10.1016/j.foreco.2007.03.047.

De Futos, P., Martínez, F. y Esteban, S. (2011). El turismo micológico como fuente de ingresos y empleo en el medio rural. El caso de Castilla y León. Estudios de Economía Aplicada, 29(1), 279-307.

Esteban, C. I. (2007). Cordyceps sinensis, un hongo usado en la medicina tradicional china. Revista Iberoamericana de Micología, 24(4), 259-262. https://doi.org/10.1016/S1130-1406(07)70052-9.

Fernández, M. V., Barroetaveña, C., Bassani, V. y Ríos, F. (2012). Rentabilidad del aprovechamiento del hongo comestible Suillus luteus para productores forestales y para familias rurales de la zona cordillerana de la provincia del Chubut, Argentina. Bosque, 33 (1), 43-52. http://dx.doi.org/10.4067/S071792002012000100005.

Franco, S., Burrola, C. y Arana, Y. (2012). Hongos silvestres comestibles: un recurso forestal no maderable del Nevado de Toluca. Toluca: UAEM.

Gardner, K. y Lewis, D. (2003). Antropología, desarrollo y el desafío posmoderno. Toluca: Colegio Mexiquense. Godet, M. (2000). La caja de herramientas de la prospectiva estratégica. Paris: Serpa.

Jasso, X., Martínez, Á. R., Gheno, Y. A. y Chávez, C. (2016). Conocimiento tradicional y vulneravilidad de hongos comestibles en un ejido dentro de un Área Natural Protegida. Polibotánica, 42, 167-196. http://dx.doi.org/10.18387/polibotanica.42.9.

Jiménez, A. E., Thomé, H. y Burrola, C. (2016). Patrimonio biocultural, turismo micológico y etnoconocimiento. Periplo sustentable, 30, 180-205.

Konu, H. (2015). Developing a forest-based wellbeing tourism product together with customers-An ethnographic approach. Tourism Management, 20, 1-16. https://doi.org/10.1016/j.tourman.2015.02.006.

Lázaro, A. (2008). El aprovechamiento micológico como vía de desarrollo rural en España: las facetas comercial y recreativa. Anales de Geografía, 28(2), 111-136.

Lara, F., Romero, A. T. y Burrolla, C. (2013). Conocimiento tradicional sobre los hongos silvestres en la comunidad otomí de San Pedro arriba; Temoaya, Estado de México. Agricultura, sociedad y desarrollo, 10(3), 305-33.

Mariaca, R., Silva, L. D. y Castaños, C. A. (2001). Procesos de recolección y comercialización de hongos comestibles silvestres en el Valle de Toluca, México. CIENCIA ergo-sum, 1(8), 30-40. 
Martínez, D. (2014). Análisis espacial de la distribución de los hongos comestibles en el Nevado de Toluca. Toluca: UAEM.

Martínez, E., Sánchez, J., Torija, R. y Vega, J. A. (2011). Turismo micológico y desarrollo sostenible del medio rural en Soria, en S. Fernández, Espacios y destinos turisticos en tiempos de globalización y crisis (332-351). Madrid: Universidad Carlos III de Madrid.

Martínez, J., Riera, P., Giergiczny, M. y Colinas, C. (2011). Value of wild mushroom picking as an environmental service. Forest Policy and Economics, 13, 419-424. https://doi.org/10.1016/j.forpol.2011.05.003.

Moreno, Á. (2002). Estudio etnomicológico comparativo entre comunidades Rarámuris de la Alta Tarabumara, en el estado de Chibuahua. Ciudad de México: UNAM.

Moreno, Á. (2013). Un recurso alimentario de los grupos originarios y mestizos de México: los hongos silvestres. Anales de Antopología, 48(1), 241-272. https://doi.org/10.1016/S0185-1225(14)70496-5.

Ortega, F. (2008). El método Delphi, prospectiva en Ciencias Sociales a través del análisis de un caso práctico. Revista Escuela de Administración de Negocios, 64, 31-54.

Salas, M. A. (2013). Prospectiva territorial. Aproximación a una base conceptual y metodológica. Mérida: Universidad de Los Andes.

Sarría, Y. y Becerra, F. Á. (2008). Aplicación del enfoque prospectivo para la gestión del desarrollo local: estudio de caso. Revista de desarrollo local sostenible, 1(2), 1-17.

Thomé, H. (2016). Turismo rural y sustentabilidad. El caso del turismo micológico en el Estado de México, en F. Carreño y A. Y. Vásquez, Ambiente y Patrimonio Cultural (pp. 43-69). Toluca: UAEM.

Toledo, C. V., Barroetaveña, C. y Rajchenberg, M. (2014). Fenología y variables ambientales asociadas a la fructificación de hongos silvestres comestibles de los bosques andino-patagónicos en Argentina. Revista Mexicana de Biodiversidad, 85, 1093-1103. https://doi.org/10.7550/rmb.40010.

Zizumbo, L., Burrola, C. y Hernández, M. (2012). El micoturismo como alternativa de desarrollo local en Amanalco de Becerra, México, en A. Cristóvão y X. Pereiro, Turismo rural em tempos de novas ruralidades (pp.825-839). Chaves: UTAD.

CC BY-NC-ND 